\title{
Double-Stranded DNA Diffusion in Slitlike Nanochannels
}

\author{
Anthony Balducci, ${ }^{\dagger}$ Pan Mao, ${ }^{\ddagger}$ Jongyoon Han, ${ }^{\S}$ and Patrick S. Doyle ${ }^{*, \dagger}$ \\ Department of Chemical Engineering, Department of Mechanical Engineering, and Department of \\ Electrical Engineering and Computer Science, Massachusetts Institute of Technology, \\ 77 Massachusetts Avenue, Cambridge, Massachusetts 02139
}

Received May 9, 2006; Revised Manuscript Received June 19, 2006

\begin{abstract}
We present an experimental study of double-stranded DNA diffusion in slitlike channels. The channel heights span the regime from moderate confinement (height $\sim$ bulk radius of gyration of the DNA) to strong confinement (height $\sim$ persistence length). Scalings of diffusivity with channel height differ from blob model predictions. The diffusivity scales inversely with molecular weight when the channel height is smaller than the bulk radius of gyration. This scaling is indicative of hydrodynamic screening. A scaling analysis shows that the screening of hydrodynamic interactions arises from a combination of two mechanisms. After using a Zimm preaverage approximation, the unique symmetry of the thin-slit disturbance velocity and the isotropic nature of the polymer conformation together cause a cancellation of hydrodynamic interactions due to symmetry. We also find that the algebraic decay of the far-field velocity magnitude is sufficient to eliminate large-length scale hydrodynamic cooperativity in diffusion of quasi-two-dimensional polymers in good solvents.
\end{abstract}

\section{Introduction}

Advances in microfabrication technologies have allowed the fabrication of fluidic channels with a characteristic dimension on the order of tens of nanometers. ${ }^{1,2}$ The potential for the use of small-channel devices in DNA mapping ${ }^{3-7}$ and separation, ${ }^{8-13}$ single biomolecule manipulation, ${ }^{12}$ and even ion separation ${ }^{14,15}$ has inspired interest in the static and dynamic response of individual molecules to confinement. ${ }^{16-18}$ The proximity of the bounding walls offers a very powerful method to change the polymer equilibrium conformation through steric interactions as well as the polymer dynamics through modulation of hydrodynamic interactions (HI). These effects manifest themselves by changing molecular transport properties such as the diffusivity, relaxation times, and mobility.

Theoretical and experimental studies of polymeric behavior in confinement extend well into the past. ${ }^{19-24}$ Studies performed in track-etched membranes examined the diffusion of polymers into and through well-defined cylindrical pores (see ref 22 for a review). These studies attempted to deconvolute the partitioning into the pore and the diffusion inside it, but the range of pore-sizes studied was ultimately restricted by the use of diffusion to force the polymer into the pore. Today, microfabrication allows the application of electric and hydrodynamic forces to transport molecules and greater control over the size and shape of the confining environment. This advantage, coupled with the use of large molecules, dramatically increases the confinement parameter space available for study. Furthermore, the use of biological macromolecules makes possible the study of monodisperse samples, and epiflouresence microscopy allows one to directly observe single DNA molecules. Austin and coworkers $^{3,4}$ have studied double-stranded (ds)-DNA behavior in small (35 to $\sim 400 \mathrm{~nm}$ ) square channels. They find expected scalings of extension with molecular weight, but scalings with channel height are slightly different from proposed theory. Also, they attribute a drastic change of relaxation behavior in channels

* Corresponding author. E-mail: pdoyle@mit.edu.

$\dagger$ Department of Chemical Engineering.

Department of Mechanical Engineering.

$\S$ Department of Electrical Engineering and Computer Science. approximately $140 \mathrm{~nm}$ square to a conformational change from a "blob-like" state ${ }^{19,21}$ to the conceptual "reflecting chain" of Odijk $^{23}$ (Figure 1). The dynamics differ greatly when the geometry is a slit, especially under strong confinement conditions. In the limiting case, a square channel or tube constricts polymer motion to a reptation mechanism, however, in a slit there is a finite lateral mobility ( $x-y$ plane in Figure 1$)$. Bakajin et al. ${ }^{18}$ studied the extension of DNA around posts in slitlike channels. They were able to prove electrohydrodynamic equivalence and showed that intramolecular HI was negligible in the smallest channel used $(90 \mathrm{~nm})$. However, the study only qualitatively reported changes to transport properties (longest relaxation time). Maier et al. ${ }^{25,26}$ studied DNA confined to a cationic lipid bilayer and found that 2-dimensional scaling laws held for both static and dynamic quantities. They also found that intramolecular HI does not affect DNA behavior due to the strong coupling between the DNA and the bilayer. Simulations have recently investigated polymer behavior in confinement. Jenderjack et al. ${ }^{27}$ used a Brownian dynamics simulation of the polymer dynamics coupled with a numerical solver for the solvent motion. In square channels, the scalings obtained did not match theoretical predictions. The authors attributed this to the importance of a length scale associated with polymerwall interactions which was unaccounted for in blob theory. The same simulations performed in slits ${ }^{17}$ have shown closer agreement with blob theory, but there still exist slight deviations. Usta et al. ${ }^{28}$ used Lattice Boltzmann simulations to study confined polymer diffusion and obtained good (visual) agreement with blob theory. However, their experiments did not consider gap height scalings in detail, as evidenced by the limited data for a given molecular weight in Figure 11 of ref 28. In simulations, as well as experiments, theoretical scalings of polymer properties with gap height have been difficult to confirm.

Recently, we studied DNA diffusion in slitlike channels made of PDMS and glass, ${ }^{17}$ but the mechanical integrity of the PDMS did not allow for study of chains confined much beyond the bulk radius of gyration. In this paper, we systematically examine the effects of channel height and molecular weight on the diffusion of ds-DNA molecules in slitlike nanochannels. It will 
(a)

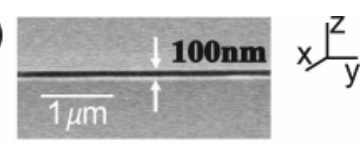

(b)

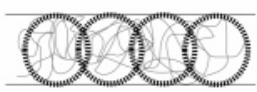

(c)

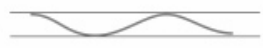

(d)

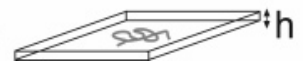

Figure 1. (a) SEM image of a glass channel cross section with a slit height of $100 \mathrm{~nm}$. Also shown are schematics of polymer conformations in a slitlike channel for (b) $R_{\mathrm{g}, \text { bulk }} \gg h \gg L_{\mathrm{p}}$ (blob regime) and (c) $h \ll$ $L_{\mathrm{p}}$ (Odijk regime). A top view (d) shows the polymer attains a 2-D random walk and is free to diffuse laterally. This motion is readily observed with fluorescence microscopy.

be shown that independent investigation of these two variables yields separate information about the conformation of the polymer and the hydrodynamics of the system, information that is lost when viewed on a single, normalized plot. While present theory relies on using the channel height as an intuitive scale for hydrodynamic screening ${ }^{19,23}$ and nearly complete hydrodynamic screening has been shown to occur when the gap height is on the order of a Kuhn length of the polymer, ${ }^{18}$ the effects of hydrodynamic interactions on diffusion in channels with gap heights near the radius of gyration of the polymer has yet to be experimentally studied. Indeed, the algebraic decay of the inplane component of point-force disturbances in quasi-twodimensions ${ }^{29}$ calls into question the use of a particular "screening" length scale, which is normally used to describe an exponential decay.

The study of polymer physics at these length scales is very important from an engineering perspective. The channel heights used here $(75-550 \mathrm{~nm})$, while easily fabricated with standard techniques, are small enough to alter the behavior of biological macromolecules substantially and in potentially very useful ways. ${ }^{1,2}$ The sizes of the DNA used in this study are comparable to those of interest in genome mapping, especially mapping based on single-molecule techniques. ${ }^{5}$ The purpose of this paper is to test applicable scaling theories for this important region of parameter space. The results of these extensive experiments also serve as a basis of comparison for current and future mesoscale computer simulations. We also present a scalinglevel analysis for the mechanisms of hydrodynamic screening in quasi-two-dimensional (Q2D) environments. The physics at these length scales is crucial to understand and these results will be important in the design of future devices to map, separate, and generally control single biomolecules for analysis.

\section{Overview}

First, we briefly review the scaling predictions for polymers in confinement. We consider a linear polymer of $N$ monomers with a persistence length $L_{\mathrm{p}}$, diameter $d$, and molecular weight $M_{\mathrm{W}} \sim N$ in a slit of height $h$. In bulk, the polymer attains a coiled configuration with a radius of gyration $R_{\mathrm{g}, \text { bulk }} \sim L_{\mathrm{p}} N^{v}$, where $v$ is the Flory-Edwards exponent $(\approx 3 / 5$ for a good solvent). ${ }^{30}$ In confinement, the polymer can be described as a chain of shorter "segments" with a characteristic length scale near $h$. The nature of these segments is defined by the static conformation the polymer can attain between the two planes. In effect, the polymer is coarse-grained near the length scale of the channel height. De Gennes and co-workers ${ }^{19,21}$ developed a theory for moderate confinement where $L_{\mathrm{p}} \ll h \ll R_{\mathrm{g} \text {,bulk }}$ and the "segments" of the polymer contain enough persistence lengths themselves to follow the high molecular weight conformational scalings developed by Flory (Figure 1b). These segments are termed "blobs" and contain $g \sim\left(h / L_{\mathrm{p}}\right)^{(1 / v)}$ monomers (there are $N_{\text {blobs }}=N / g$ blobs per chain). For stronger confinement $\left(h \ll L_{\mathrm{p}}\right)$, the chain cannot double back on itself and attains a "reflecting" conformation (Figure 1c) as described by Odijk. ${ }^{23}$ In this case, we take the polymer segment to be a rod with length equal to the Odijk length scale $\left(\lambda_{\text {Odijk }} \sim\right.$ $\left.L_{\mathrm{p}}{ }^{1 / 3} h^{2 / 3}\right)^{23,31,32}$ and diameter $d$ (there are $N_{\text {rods }} \sim N / \lambda_{\text {Odijk }}$ rods per chain). In the development of these theories, it is assumed that cooperative motion of the segments due to $\mathrm{HI}$ is negligible because $\mathrm{HI}$ is screened over distances larger than the channel height. The drag on the chain is then solely dependent on the drag on a polymer segment and the number of those segments in the chain $\left(\xi_{\text {chain }} \sim x_{\text {segment }} N_{\text {segment }}\right)$. The drag on an individual segment is taken to be $\xi_{\text {blob }} \sim h^{19,21}$ and $\xi_{\text {rod }} \sim \lambda_{\text {Odijk }} / \log (h /$ $d)^{3,33}$ for the blobs and rods, respectively. Taking into account both the conformation and the hydrodynamics, scaling expressions for the in-plane diffusivity ( $\left.D \sim 1 / \xi_{\text {chain }}\right)$ in good solvent are the following

$$
\begin{gathered}
D_{\text {blob }} \sim \frac{1}{\xi_{\text {blob }} N_{\text {blobs }}} \sim\left[\frac{1}{h}\right]\left(\frac{h^{5 / 3}}{M_{\mathrm{w}} L_{\mathrm{p}}^{5 / 3}}\right) \\
D_{\text {Odijk }} \sim \frac{1}{\xi_{\text {rod }} N_{\text {rods }}} \sim\left[\frac{\ln (h / d)}{\lambda_{\text {Odijk }}}\right]\left(\frac{\lambda_{\text {Odijk }}}{M_{\mathrm{w}}}\right)
\end{gathered}
$$

The equations are left expanded to emphasize the contribution of the drag on an individual segment (square brackets), the number of segments defined by the conformation between the planes (parentheses), and the screened HI (unity exponent on the term in parentheses). Note that scaling of $D$ with $M_{\mathrm{w}}$ isolates the effect of hydrodynamic interactions. The scaling of $D$ with $h$ is a combined effect of hydrodynamics, the conformation of a segment, and the drag on that segment. Also, since hydrodynamics are assumed to be screened regardless of the shape of the polymer, we can expect $D \sim M_{\mathrm{w}}{ }^{-1}$ even for channel heights between the ranges of applicability of the two equations above.

While making the assumption of screened hydrodynamic interaction simplifies the analysis, a closer examination calls the assumption into question. Hydrodynamic interaction occurs through disturbances of the solvent, the velocity of which must satisfy Stokes' equations at low Reynolds numbers. For the case of the far-field velocity of a point force between two parallel planes, the expression obtained by Liron and Mochon ${ }^{29}$ can be reduced to

$$
v(\boldsymbol{r}) \sim \frac{1}{|\boldsymbol{r}|^{2}}\left(\hat{\boldsymbol{r}} \hat{\boldsymbol{r}}-\frac{1}{2} \boldsymbol{I}\right) \cdot \boldsymbol{f}
$$

where $\boldsymbol{I}$ is the unit tensor and $v$ is the in-plane velocity at a point defined by the vector $\boldsymbol{r}(\hat{\boldsymbol{r}}=\boldsymbol{r} /|\boldsymbol{r}|)$ due to a point force $\boldsymbol{f}$ at the origin. This expression is valid for $|\boldsymbol{r}|>h$ and decays only slightly faster than in bulk $(1 /|\boldsymbol{r}|)$. The rate of this algebraic decay alone has never been tested (to our knowledge) to be able to dampen the disturbance enough to eliminate cooperative motion between polymer segments, although its orientational effects have been studied in the HI between colloids. ${ }^{34-37}$

\section{Experimental Section}

3.1. Channel Fabrication. We experimentally observe doublestranded DNA using single molecule epifluorescence microscopy in slitlike nanochannels with gap heights between 75 and $545 \mathrm{~nm}$ and a width of $150 \mu \mathrm{m}$. The glass nanochannels used in this study were fabricated as described in Mao and Han. ${ }^{2}$ A schematic of the 
Table 1. Molecular Weight Dependent Properties of Stained DNA ${ }^{a}$

\begin{tabular}{lccl}
\hline \multicolumn{1}{c}{ DNA } & basepairs $\times 10^{-3}$ & $D_{\text {bulk }}\left(\mu \mathrm{m} / \mathrm{s}^{2}\right)$ & $R_{\text {g,bulk }}(\mu \mathrm{m})$ \\
\hline M13mp18 & 7.2 & $1.32 \pm 0.1$ & $0.24 \pm 0.02$ \\
$1 / 2 \lambda$ & $24.4 \pm 0.2$ & $0.65 \pm 0.06$ & $0.45 \pm 0.04$ \\
$\lambda$ & 48.5 & $0.46 \pm 0.03$ & $0.69 \pm 0.05$ \\
$2 \lambda$ & 97.0 & $0.28 \pm 0.07$ & $1.15 \pm 0.03$ \\
T4 & 165.6 & $0.22 \pm 0.03$ & $1.46 \pm 0.2$
\end{tabular}

${ }^{a}$ Bulk diffusivity and radius of gyration data for T4 DNA was obtained through interpolation of data in Smith et al. ${ }^{39}$ since we could not obtain significantly long enough traces where the entire molecule was in focus for the bulk measurement. Radii of gyration are obtained from the bulk diffusivity via $R_{\text {g,bulk }}=0.203 k_{\mathrm{b}} T /\left(\sqrt{6} \eta D_{\text {bulk }}\right)$.

geometry and an SEM image of a channel is given in Figure 1. The channels are found to have low surface roughness without appreciable sagging. ${ }^{2}$ We observe the DNA at least $15 \mu \mathrm{m}$ away from the side of the channel to minimize any hydrodynamic effects related to the sides of the channel. Diffusivities calculated in channels as narrow as $50 \mu \mathrm{m}$ wide do not vary appreciably from the data reported here.

3.2. DNA Preparation. $\lambda$-DNA ( $48.5 \mathrm{kbp})$ and M13mp18 (7.2 kbp) DNA were purchased from New England Biolabs (NEB) and T4GT7 (165.6 kbp) DNA from Nippon Gene. An XbaI (NEB) digestion was used to linearize the M13mp18 DNA and cleave the $\lambda$-DNA at the 24508 th basepair, resulting in two $\lambda$-DNA halfmers $(1 / 2 \lambda)( \pm 250 \mathrm{bp})$. $\lambda$-DNA dimers $(2 \lambda)$ were made using an oligoprotected ligation ${ }^{38}$ (DNA oligomers from Sigma Genosys). The results of the biomolecular techniques were tested by staining and then combing the DNA onto a polystyrene-coated coverslip and measuring their contour length relative to a $\lambda$-DNA control. This

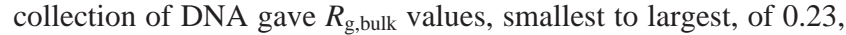
$0.49,0.69,1.15$, and $1.48 \mu \mathrm{m}$ as calculated from bulk diffusion measurements via $R_{\mathrm{g}, \text { bulk }}=0.203 k_{\mathrm{b}} T /\left(\sqrt{6 \eta} D_{\text {bulk }}\right){ }^{17,39}$ The DNA properties are summarized in Table 1.

Experimental runs were carried out in $0.5 \times$ TBE $(0.045 \mathrm{M}$ TrisBorate, $1 \mathrm{mM}$ EDTA, OmniPur) and 4 vol $\% \beta$-mercaptoethanol (BME) (CabioChem). The viscosity of this buffer was $1.1 \mathrm{cP}$, measured through a diffusion analysis of $0.518 \mu \mathrm{m}$ (diameter) spherical beads. The Debye length in this system is $\approx 2.5 \mathrm{~nm}$, much less than the smallest channel height used. Repulsive electrostatic interactions with the channel surfaces are not expected to play a large role in processes other than to confine the DNA to the channel. Since the ligation and digestion reactions were carried out in different buffers (NEB), exchange to the TBE buffer took place in Centricon (Millipore) filters. We found we could not use TBE buffer directly in the Centricons, presumably due to an interaction between the borate ion, the cellulose filter, and the DNA, ${ }^{40}$ so the exchange was first to TE buffer [0.09 M Tris (Promega), 2 mM EDTA (OmniPur)] and then diluted with equal parts in $0.09 \mathrm{M}$ boric acid (J. T. Baker). DNA was stained with YOYO-1 dye (Molecular Probes) at a 4:1 base pair to dye molecule ratio (saturation) and allowed to sit overnight. Directly before use, the channel was loaded with the TBE/BME buffer (without DNA) and an electric field was applied to induce electroosmotic flow and equilibrate the channel. DNA-containing buffer was then placed at the cathodic reservoir and loaded (typical field $\sim 100 \mathrm{~V} / \mathrm{cm}$ ) into the channel for observation. Bulk measurements were obtained in a custom diffusion cell consisting of two glass slides separated by a $\sim 100$ $\mu \mathrm{m}$ Parafilm spacer and sealed with epoxy.

3.3. Observation and Analysis. Epifluorescence microscopy was used to observe the DNA using a set up described previously. ${ }^{17}$ Images of the stained DNA were collected with a Hamatsu EBCCD camera (model: 7190-21) at 30 frames per second. Image analysis was performed using custom-written code and IDL software. In each frame, the background noise level was subtracted from the image and then the DNA center of mass (in the $x-y$ plane) was calculated as the first moment of the intensity distribution ${ }^{17,41}$ (see Figure 2). The centers of mass of more than 20 DNA were tracked for an average of $10 \mathrm{~s}$ each for every channel height and molecular weight condition. We calculated the center of mass displacement as a function of the time elapsed between the frames in which the displacement was measured (lagtime, $\tau$ ). The gapaveraged, in-plane diffusion coefficient $(D)$ was obtained from the slope of the mean-square displacement (MSD) of the ensemble vs lagtime. ${ }^{17}$ To obtain an estimate for the error in our study, we divide our experiments into smaller ensembles each consisting of a single DNA molecule. The diffusivity calculated for each individual molecule is an averaged property of the many displacements and lagtimes in each trajectory. The problem, then, is finding the standard deviation of all of our measurements from the means of a number of samples. The standard deviation in the average diffusivity over many DNA molecules can be calculated as $\sigma_{\mathrm{D}} /$ $\sqrt{N_{\text {DNA }}}$ where $\sigma_{\mathrm{D}}$ is the standard deviation of the diffusivities of individual DNA molecules and $N_{\mathrm{DNA}}$ is the number of DNA molecules (tracked for a constant amount of time). ${ }^{42}$ This result is found to be consistent with the difference between diffusivities calculated in the independent $x$ and y dimensions and is on the same order as the value found through error propagation of the uncertainty in the variance of the displacements. We calculated the probability density functions of the displacement at all lagtimes (van Hove correlation functions) to ensure the absence of appreciable flow effects (see Figure 2). Also, the probability density functions were normally distributed and the mean-square displacement plots were linear, implying a lack of adsorption to the channel walls and/or obstruction in the channels over observable time scales. ${ }^{43}$ In addition, the conformation of the DNA during loading and unloading into the visualization area did not show evidence of stretching or deformation due to interactions with the surface.

\section{Results and Discussion}

4.1. Nanoslit Experimental Results. Experimental results of confined behavior are plotted on a master curve to test the applicability of blob theory. If blob theory holds, a plot of the in-plane diffusivity in the confined slit $(D)$ normalized by the diffusivity in bulk $\left(D_{\text {bulk }}\right)$ vs $R_{\mathrm{g}, \text { bulk }} / h$ yields a universal curve with a slope of $-2 / 3 .{ }^{17,21}$ Figure 3 shows this plot for all of the data collected in this study compared with results from Chen et al. ${ }^{17}$ Comparison with simulation and prior experiment is very favorable. Most of the information we can extract from this plot comes in the form of the data collapse. Here, the collapse is good, although the spread of the band is outside of the error bars (1 standard deviation). Without separately analyzing the trends with channel height and molecular weight, it is impossible to know if the spread is due to experimental scatter or systematic deviation. By independently examining scalings of diffusivity with molecular weight and gap height, we are able to determine which assumptions used in scaling theories hold for this system and the regions where they break down.

Figure 4 shows diffusivity as a function of channel height for different DNA molecular weights. For complete curve collapse in Figure 3, the slopes in Figure 4 must be constant. In Figure 4 we see that the slopes systematically increase with increasing molecular weight. For the two largest DNA ( $\lambda$ and $2 \lambda$ ), the slopes are $0.55 \pm 0.05$ and $0.55 \pm 0.06$, respectively. These slopes should be compared to the blob theory scaling of 0.67. This difference is shown more clearly in Figure 5. Here, we compare the diffusivity divided by the gap height raised to powers predicted by blob theory (Figure 5a) and found through the fit in Figure 4 (Figure 5b), both normalized by the value found in the $545 \mathrm{~nm}$ channel. We see small but systematic deviation from unity when comparing to blob theory, and that this deviation decreases with increasing molecular weight. Indeed, for the $2 \lambda$ DNA, the deviations are of the same order in the two plots, but the residuals in Figure 5a are systematically positive. To better understand this we must consider the limitations of blob theory. The bounds of the applicability of 
(a)
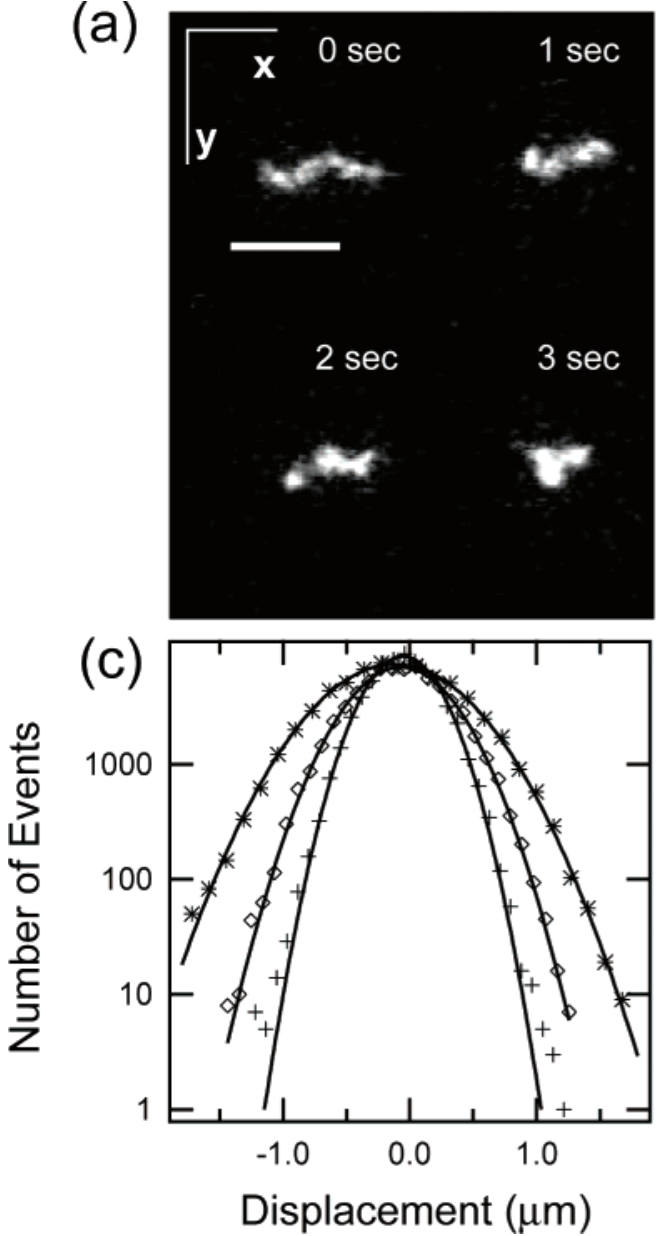

(b)

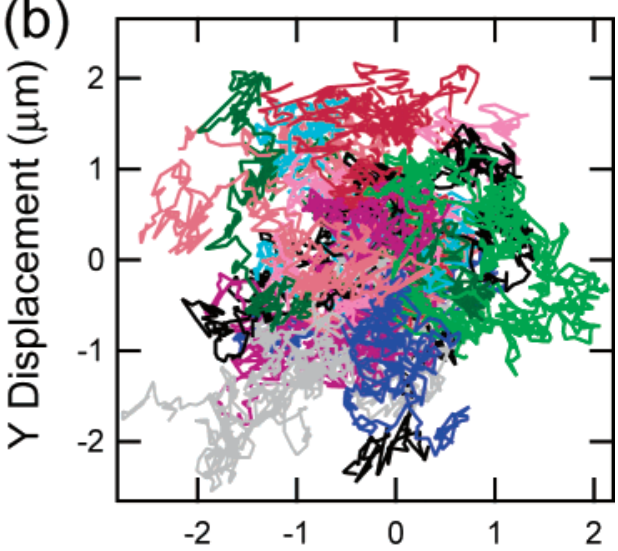

X Displacement $(\mu \mathrm{m})$

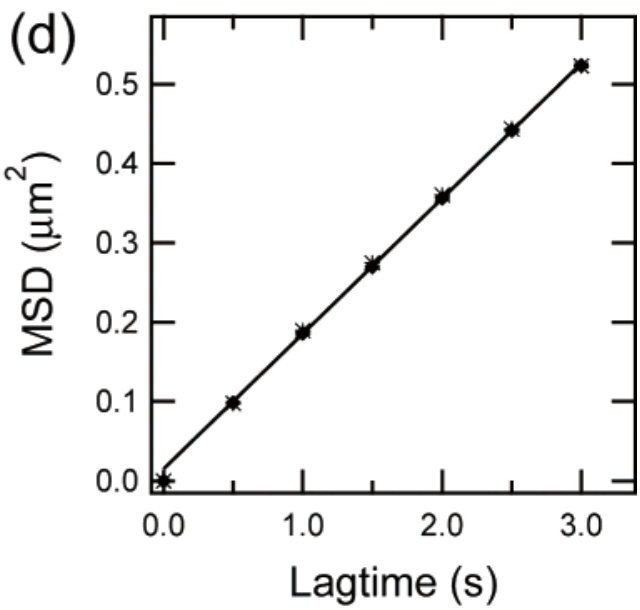

Figure 2. Summary of image analysis for $2 \lambda$-DNA in a $545 \mathrm{~nm}$ tall channel. (a) Time-series images from camera over four seconds $(\Delta t=1 \mathrm{~s})$ of a DNA movie (scale bar $=5.0 \mu \mathrm{m}$ ). (b) Center of mass trajectories for $282 \lambda$-DNA molecules. (c) Probability density functions (not normalized) for the trajectories at a lagtime of $0.33(+), 0.66(\diamond)$, and $1.23(*)$ s with accompanying fits to a Gaussian curve (solid lines). (d) Mean-squared displacement $(\mathrm{MSD})(\diamond)$ and the variance of the probability density function $(*)$ as a function of lagtime. The two are identical in the limit of no net flow in the system. The MSD is fit to a line between lagtimes of 0.5 and $1.5 \mathrm{~s}$ (with a data point every $1 / 30 \mathrm{~s}$ ), where short-time error associated with small displacements and the long time error associated with poor statistical information are minimized. The nonzero $y$-intercept of the fit to the MSD (solid line) is related to the image signal-to-noise. ${ }^{62}$

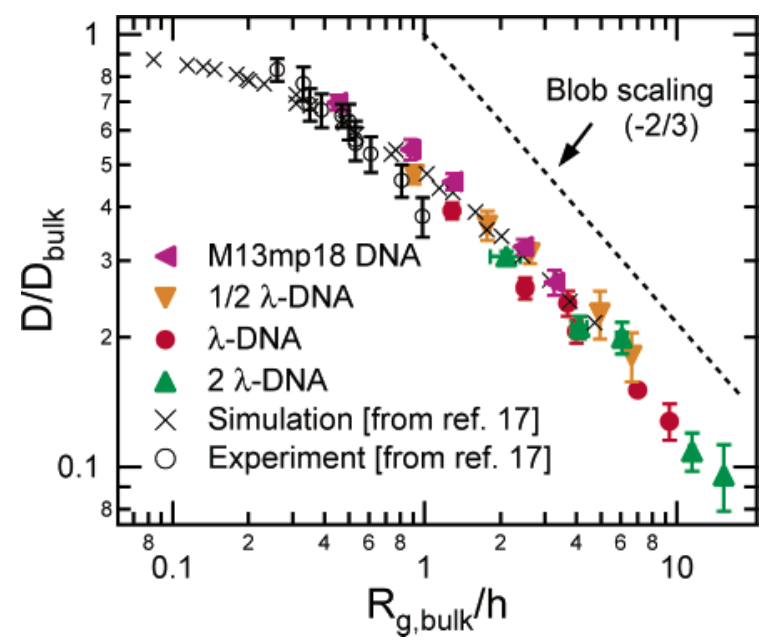

Figure 3. Curve collapse for all data plotted on normalized axes. Diffusivity is normalized by the bulk diffusivity, and the channel height by the bulk radius of gyration of the DNA. Also included is the simulation $(x)$ and experimental $(O)$ data from Chen et al. ${ }^{17}$

blob theory are set by the ability of the polymer to form blobs on the length scale set by $h$ (which sets a lower limit on $h$ ) and having sufficient confinement of the polymer (which sets a lower limit on $M_{\mathrm{w}}$ and an upper limit on $h$ ). Experimentally, Reisner

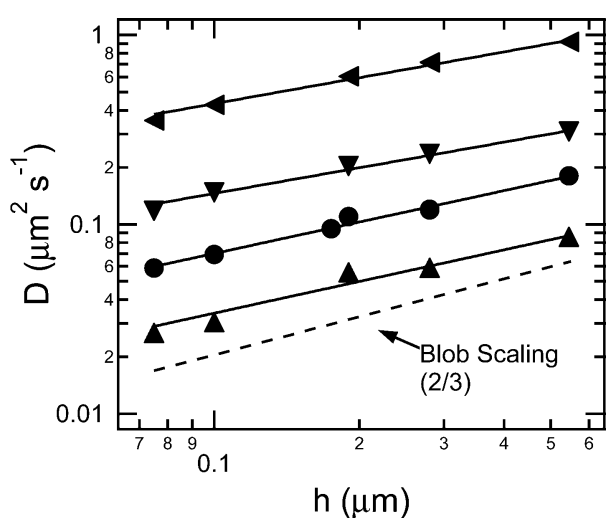

Figure 4. Diffusivity vs channel height for different molecular weights. Solid lines are fits to the data, the dotted line is the slope expected for blob theory, and the error is less than the size of the symbols. The symbols correspond as follows: solid triangle pointing left, M13mp18 DNA; $\boldsymbol{\nabla}, 1 / 2 \lambda$-DNA; $\bullet$ DNA; $\mathbf{\Lambda}, 2 \lambda$-DNA. The respective slopes are $0.45 \pm 0.03,0.45 \pm 0.04,0.55 \pm 0.05$, and $0.55 \pm 0.06$.

et al. $^{3}$ found that there was a drastic change in scalings of extension and relaxation times in channels approximately 140 $\mathrm{nm}$ square. They attribute the change in scalings to the onset of the Odijk regime, effectively setting the lowest conceivable bound on the channel heights to which blob theory can be applied for ds-DNA. Strikingly, this distance is only about twice 
(a)
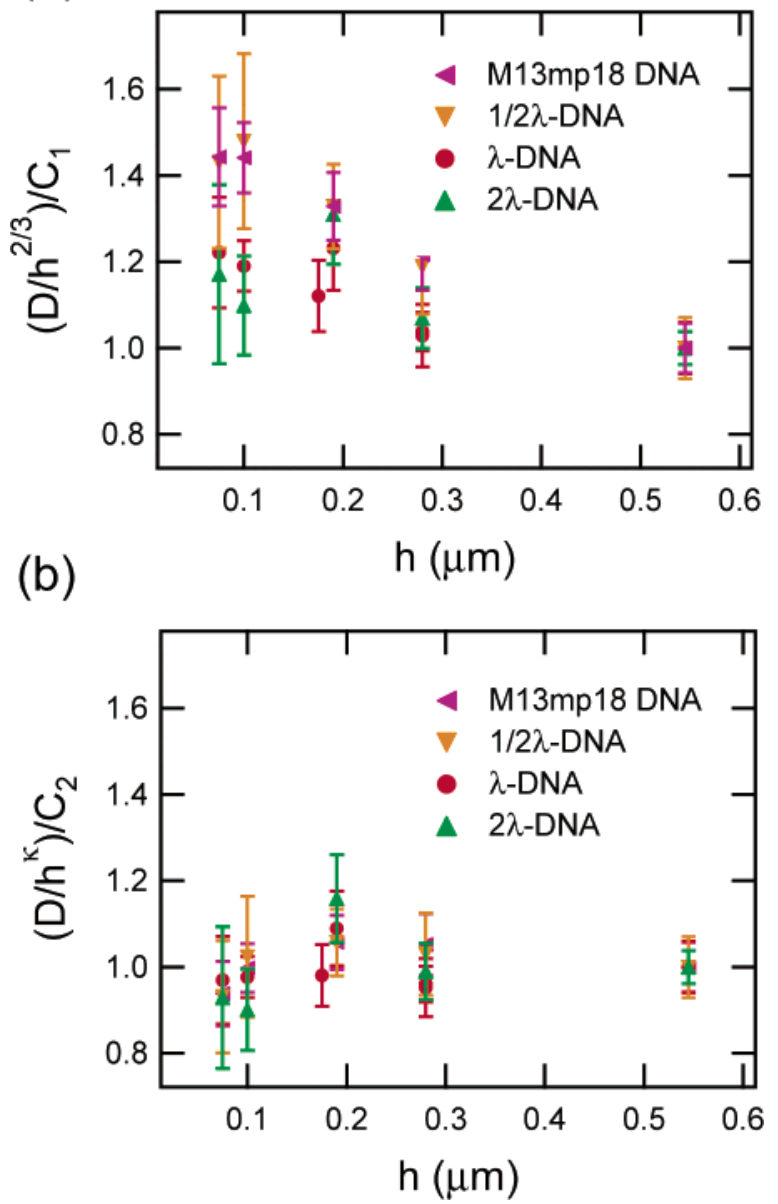

Figure 5. Tests of power law fits. (a) Diffusivity divided by the gap height to the $2 / 3$, normalized by the value obtained in the $545 \mathrm{~nm}$ channel $\left(C_{1}\right)$. If blob theory holds, these values should be constant at unity. However, we see small but systematic deviations. (b) Diffusivity divided by the gap height raised to the power found in the fits in Figure $4(\kappa)$, normalized by the value obtained in the $545 \mathrm{~nm}$ channel $\left(C_{2}\right)$.

the persistence length, which, when combined with the observed scalings for larger channels in ref 3, brings into question whether the polymer is in a true blob-like conformation. In channels slightly larger than this bound, the blobs may be too small to contain enough chain to follow the long-chain scalings of blob size with the number of monomers due to excluded volume $(\mathrm{EV})$ effects. ${ }^{44,45}$ In this regime, the number density of monomers within each blob is small enough that the free energy increase due to internal repulsions of the chain is less than the thermal energy. Hence, the $g$ dependence ( $N$ dependence in a coil) of the second-virial term accounting for these interactions in Flory scaling theory is overestimated and scalings of the blob size with $g$ appear to be closer to $\Theta$-solvent scalings. ${ }^{45}$ The concept of the "thermal blob" 45 is used to define the polymer size where the effects of EV can be considered to be in the long chain limit. For ds-DNA in our buffer conditions, this length scale (in terms of the radius of gyration of the thermal blob) is approximately $\xi_{T}=4 L_{\mathrm{p}}{ }^{2} / \sqrt{6} d \approx 0.8 \mu$ m where we use $L_{\mathrm{p}} \approx 50$ $\mathrm{nm}$ and $d \approx 5 \mathrm{~nm}$ (twice the Debye length for our buffer conditions). ${ }^{44,45}$ This distance is larger than the channel heights investigated here, and may lead to different experimental scalings due to the fact that the internal structure of a blob is not correctly described in the theory (i.e., $1 / 2<v(g)<3 / 5$ and not $\approx 3 / 5$ as assumed) especially in the smaller channels.

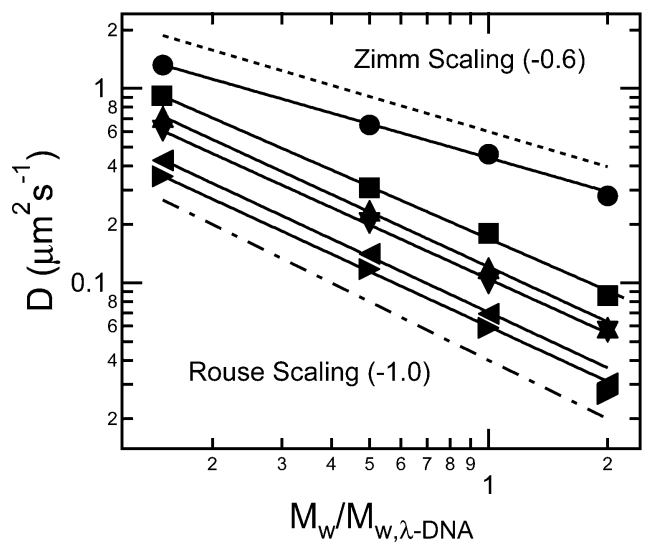

Figure 6. Diffusivity vs molecular weight $\left(M_{\mathrm{w}}\right)$ relative to that of $\lambda$-DNA $\left(M_{\mathrm{w}, \lambda \text {-DNA }}\right)$. Solid lines are fits to the data, the dotted line is the scaling expected for Zimm hydrodynamics, the dash-dot line is the scaling expected for Rouse hydrodynamics. The symbols correspond as follows: $\boldsymbol{Q}$, bulk; $\mathbf{\square}, 545 \mathrm{~nm} ; \boldsymbol{\Lambda}, 280 \mathrm{~nm} ; \boldsymbol{\nabla}, 190 \mathrm{~nm}$; solid triangle pointing left, $100 \mathrm{~nm}$; solid triangle pointing right, $75 \mathrm{~nm}$. The respective slopes are the following: $-0.57 \pm 0.01,-0.85 \pm 0.04,-0.93$ $\pm 0.05,-0.93 \pm 0.06,-0.95 \pm 0.07$, and $-0.94 \pm 0.07$.

Incorporation of these short chain effects on EV causes an increase in the theoretical slope of $\log (D)$ against $\log (h)$, which does not agree with our data. Setting $v=0.5$ in the scaling of relaxation times and extensions with channel height, however, brings the theoretical scalings closer to the experimental results of Reisner et al. ${ }^{3}$ We propose that there is a rather large parameter space between the blob and Odijk regimes. This transition regime is practically important because channels in this region are both easily fabricated and can confine DNA molecules with sizes of interest for genomic mapping technologies.

The other bound on the applicability of blob theory ensures that the polymer is moderately confined by setting the ratio of maximum channel height to minimum molecular weight. Hsu et al. ${ }^{46}$ showed using Monte Carlo simulations that the "blob" conformation scalings cannot be expected to hold until $R_{\mathrm{g}, \mathrm{bulk}} / h$ $z 2$. We find, then, that the data collected in this study only brushes blob theory parameter space. The remaining assumption that the walls do not increase the drag on individual blobs should be examined if future experimental results cannot be brought into line with the theory. ${ }^{27,47}$ Also important to note, in both Figures 3 and 4, there is no dramatic change in trend at smaller channel heights even though our channels are in the range predicted for the onset of the Odijk regime. ${ }^{3}$ This implies the transition to the Odijk regime is smooth for diffusion in slitlike channels (unlike relaxation in square channels). ${ }^{3}$

Shown in Figure 6 is diffusivity vs molecular weight for varying channel heights and bulk measurements. The bulk data and trend agree well with previous measurements. ${ }^{17,39}$ The molecular weight is normalized with that of $\lambda$-DNA as a convenient scaling factor and since $\lambda$-DNA has become a standard in single-molecule DNA studies. The scaling of diffusivity with molecular weight approaches Rouse scaling $(-1.0)$ as the channel height is decreased, indicating hydrodynamic screening over the coil length of the polymer, in agreement with scaling theory (i.e., the exponents on $M_{\mathrm{w}}$ in eq 1 and eq 2 are indeed unity). This is the first experimental evidence showing that the effects of $\mathrm{HI}$ are negligible in describing coil behavior at channel heights only slightly smaller than the bulk radius of gyration of the polymer.

To investigate the crossover to Rouse-like behavior further, we chose a channel with a gap height $(h=545 \mathrm{~nm})$ intermediate 
to the radii of gyration of available DNA and extended the molecular weight range with T4 DNA (see Figure 7). We compare the diffusion of DNA to an expression for the gapaveraged, in-plane diffusivity of a sphere between two plane walls. $^{48}$

$$
\begin{aligned}
& \frac{D}{D_{\text {bulk }}}= \\
& \frac{\left(\begin{array}{c}
1+\frac{9}{16}\left(\frac{2 R_{\mathrm{h}}}{h}\right) \ln \left(\frac{2 R_{\mathrm{h}}}{h}\right)-1.19358\left(\frac{2 R_{\mathrm{h}}}{h}\right)+ \\
0.4285\left(\frac{2 R_{\mathrm{h}}}{h}\right)^{3}-0.3192\left(\frac{2 R_{\mathrm{h}}}{h}\right)^{4}+0.08428\left(\frac{2 R_{\mathrm{h}}}{h}\right)^{5}
\end{array}\right)}{\left(1-\frac{2 R_{\mathrm{h}}}{h}\right)}
\end{aligned}
$$

where $R_{\mathrm{h}}$ is the hydrodynamic radius of the DNA defined by setting the Stokes-Einstein diffusivity equal to the Zimm diffusivity: $D_{\text {bulk }}=\left(k_{\mathrm{b}} T\right) /\left(6 \pi \eta R_{\mathrm{h}}\right)=\left(0.203 k_{\mathrm{b}} T\right) /\left(\sqrt{6} \eta R_{\mathrm{g}, \text { bulk }}\right)$. This expression has been shown to be in excellent agreement with experimental results of spherical colloids in slit confinement, even in highly confining environments (i.e., $2 R_{\mathrm{h}} / h z$ $0.8) .{ }^{48}$ We compare to the diffusivity of a sphere because, in the large-channel limit, the hydrodynamic disturbance caused by the moving DNA molecule can be assumed to be in the farfield limit at the channel walls. In this case, information about the local structure of the DNA is lost and the disturbance is that of a sphere of radius $R_{\mathrm{h}}$. Here, we can view the DNA as an effective sphere (i.e., coarse-grained to a single bead), and examine the increased drag on that sphere due to the presence of the walls. However, we see in Figure 7 that the expression falls well below the observed diffusivity of DNA. This underestimation could be due to the fact that the steric length scale of the polymer is greater than its hydrodynamic length scale $\left(R_{\mathrm{g}}>R_{\mathrm{h}}\right)$. This would tend to locate the DNA closer to the center of the channel than accounted for in eq 4 . To check this, we compare the diffusion of the DNA to the diffusivity of a sphere midway between the two planes with the Faxén centerline approximation. This expression yields an upper bound for the diffusivity of a sphere in a slit channel. ${ }^{49}$

$$
\begin{aligned}
& \frac{D}{D_{\text {bulk }}}=1-1.004 \frac{2 R_{\mathrm{h}}}{h}+ 0.418\left(\frac{2 R_{\mathrm{h}}}{h}\right)^{3}+ \\
& 0.210\left(\frac{2 R_{\mathrm{h}}}{h}\right)^{4}-0.169\left(\frac{2 R_{\mathrm{h}}}{h}\right)^{5}
\end{aligned}
$$

Since the derivation of this equation assumes far-field hydrodynamics, its applicability at high confinement is limited. For the smallest DNA in our study, $R_{\mathrm{h}} / h \approx 0.5$ is still in the range where the centerline approximation is expected to hold, but the diffusivity is still slightly greater than predicted by eq 5 . However, at low molecular weight, the overall trend tends toward spherelike behavior.

As the molecular weight is increased and the DNA becomes more confined, diffusion follows Rouse scalings for polymers larger than $\lambda$-DNA. Using scaling arguments (i.e., $R_{\mathrm{g}, \text { bulk }} \sim$ $\left.M_{\mathrm{w}}{ }^{0.6}\right)$, the relative molecular weight where the channel height is equal to the bulk radius of gyration is $M_{\mathrm{w}} / M_{\mathrm{w}, \lambda-\mathrm{DNA}} \approx 0.7$. To the precision afforded by the DNA used here, we find that for all chains where $R_{\mathrm{g}, \text { bulk }}>h$, diffusivity scales linearly with molecular weight for a fixed value of $h$. This scaling directly confirms that intramolecular hydrodynamic interactions do not give rise to cooperative behavior over length scales governing molecular diffusion.

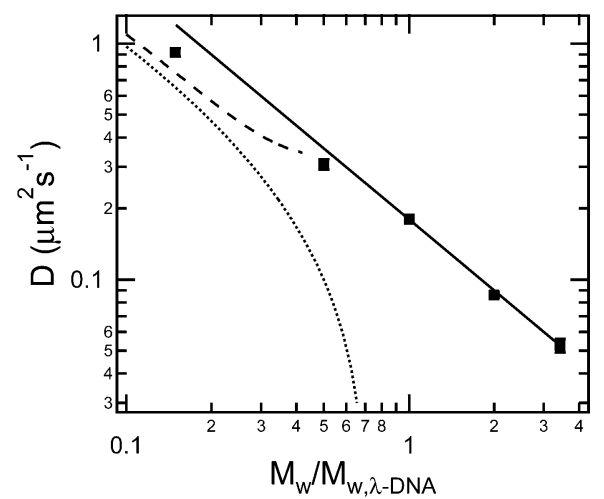

Figure 7. Diffusivity vs relative molecular weight in a $545 \mathrm{~nm}$ channel (points). The relative molecular weight where $R_{\mathrm{g}, \mathrm{bulk}}=h$ is $M_{\mathrm{w}} / M_{\mathrm{w}, \lambda-\mathrm{DNA}}$ $\approx 0.7$. Included are expressions for the gap-averaged, in-plane diffusion of a sphere in a slit pore (eq 4, dotted line), the local, in-plane diffusion of a sphere at the centerline of the channel (eq 5, dashed line), and Rouse scalings (solid line). The diffusivity at the centerline is plotted to the relative molecular weight where $2 R_{\mathrm{h}} / h \approx 1$, which already pushes the applicability of the theory, but after which there is a nonphysical upturn. DNA behavior smoothly transitions to free-draining behavior when $R_{\mathrm{g}, \mathrm{bulk}} \approx h$.

4.2. Hydrodynamic Screening Scaling Analysis. Recently, colloidal experiments on the hydrodynamics in confined environments have yielded surprising results. ${ }^{35,37}$ The HI was found to have an inverse (or anti-drag) coupling in the transverse direction, originating from the far-field dipolar flow incurred by a point force in slit confinement. ${ }^{29}$ Pair interactions were also found to be measurable over distances as far as an order of magnitude larger than the particle radius when the particle size was a substantial fraction of the channel height. ${ }^{35,37}$ It has also been shown in Q2D environments ${ }^{34}$ that the average hydrodynamic force on a particle due to interparticle HI vanishes if the suspension is isotropically distributed. The complex and long-ranged interactions between colloids in slit confinement seem paradoxical when compared with the evidence of hydrodynamic screening presented here, and it is worthwhile to bring the two results into line.

We now present an argument for the scaling of the longrange hydrodynamic interactions with molecular weight. Our goal in this derivation is a general expression that can be used to determine whether hydrodynamic interaction is important at the length scale of the coil of a given polymer. The parameters in the expression are the rate of the algebraic velocity decay of the solvent due to a point force disturbance as well as the density of hydrodynamic interactions (i.e., the monomer distribution in space). These parameters are governed by both the geometry and the solvent quality. The final equation has been presented before in relation to electrophoresis, ${ }^{50}$ but the details of the derivation that provide its applicability to a variety of systems of interest are given below. The analysis will compare the drag on the chain induced by HI between chain segments and the single body effect of the drag on each segment. We evaluate the expression at the coil radius to determine whether HI effects can be neglected at that length scale. If so, center of mass diffusion is expected to follow Rouse scalings. ${ }^{51}$

In the derivation that follows we will first consider, for the sake of an example, a bead-spring model of a Gaussian chain at equilibrium in Q2D. We assume the confined dimension is small enough that the conformation of the coarse-grained chain is strictly two-dimensional, and we use the $\mathrm{HI}$ interaction tensor for a Q2D geometry. This can be simulated, for instance, with modified spring forces that take into account the restricted phase space of the polymer and the pertinent length scales in 
confinement. ${ }^{52,53} \mathrm{We}$ will use the convention where a tensor is denoted by an upper case letter in bold face font and a vector by a lower case letter in bold face font. For a coarse-grained model, the motion of the chain is described by the Langevin equation $^{51}$

$$
\frac{\partial \boldsymbol{r}_{n}}{\partial t}=\sum_{m=1}^{N} \boldsymbol{H}_{n m} \cdot\left(\boldsymbol{f}_{m}^{\mathrm{B}}+\boldsymbol{f}_{m}^{\mathrm{T}}+\boldsymbol{f}_{m}^{\mathrm{E}}\right)
$$

where $\boldsymbol{r}_{n}$ is the position of bead $n, t$ is time, $N$ is the total number of beads, $\boldsymbol{H}_{n m}$ is the hydrodynamic interaction tensor, and $\boldsymbol{f}_{m}^{\mathrm{B}}$, $\boldsymbol{f}_{m}^{\mathrm{T}}$, and $\boldsymbol{f}_{m}^{\mathrm{E}}$ are the Brownian, tension, and external forces, respectively, on bead $m$. The tensor $\boldsymbol{H}_{n m}$ propagates those forces through the solvent and into a velocity of bead $n$. As mentioned above, since the interaction occurs through disturbances in the solvent, at far enough distances from the disturbance the form of the interaction must follow the far-field flow solutions for a point force. Here, we consider a Q2D geometry, where the third dimension is small compared to the radius of gyration of the polymer and can be coarse-grained into the bead-spring model. The form of the interaction tensor over distances greater than the channel height is of the form

$$
\boldsymbol{H}_{n m} \sim \frac{1}{\left|\boldsymbol{r}_{n m}\right|^{2}}\left(\hat{\boldsymbol{r}}_{n m} \hat{\boldsymbol{r}}_{n m}-\frac{1}{2} \boldsymbol{I}\right)\left(1-\delta_{n m}\right)+\boldsymbol{A}_{n} \delta_{n m}
$$

where $\boldsymbol{r}_{n m}$ is the vector from bead $n$ to bead $m, \hat{\boldsymbol{r}}_{n m}$ is a unit vector in the direction of $\boldsymbol{r}_{n m}, \delta_{n m}$ is the Kronecker delta function, and $\boldsymbol{I}$ is the identity tensor. $\boldsymbol{A}_{n}$ is the self-mobility tensor of a single bead and would presumably depend on the position of the bead relative to the walls and the gap height. ${ }^{49}$ In eq 6 , we implicity use the linearity of the Stokes' equations to add the effects of the velocity disturbances from all of the other beads, resulting in a set of nonlinear and coupled equations. We therefore use a Zimm preaveraging approximation ${ }^{51} \boldsymbol{H}_{n m} \Longrightarrow$ $\boldsymbol{H}_{n m, \text { eq }}$, where

$$
\boldsymbol{H}_{n m, \mathrm{eq}}=\int_{0}^{\infty} \int_{0}^{2 \pi} \boldsymbol{H}_{n m}\left(\left|\boldsymbol{r}_{n m}\right|, \theta\right) \Psi\left(|n-m|,\left|\boldsymbol{r}_{n m}\right|, \theta\right) \mathrm{d}\left|\boldsymbol{r}_{n m}\right| \mathrm{d} \theta
$$

and $\Psi$ is the two-dimensional Gaussian probability distribution function for the distance between two beads separated by $\mid n-$ $m \mid$ beads along the chain. We write the expression in polar coordinates to emphasize the orientational dependence of the tensor. After splitting the integral into the magnitude and orientational parts, we obtain

$$
\begin{array}{r}
\boldsymbol{H}_{n m, \mathrm{eq}} \sim \\
\int_{0}^{\infty} \frac{1}{\left|\boldsymbol{r}_{n m}\right|^{2}}\left(\frac{3}{2 \pi|n-m| L_{p}^{2}}\right) \exp \left(\frac{-\left|\boldsymbol{r}_{n m}\right|^{2}}{2|n-m| L_{p}^{2}}\right)\left|\boldsymbol{r}_{n m}\right| \mathrm{d}\left|\boldsymbol{r}_{n m}\right| \times \\
\int_{0}^{2 \pi}\left(\hat{\boldsymbol{r}}_{n m} \hat{\boldsymbol{r}}_{n m}-\frac{1}{2} \boldsymbol{I}\right) \mathrm{d} \theta\left(1-\delta_{n m}\right)+\boldsymbol{A}_{n} \delta_{n m}
\end{array}
$$

We note that the integral over $\theta$ vanishes due to the symmetry of the interaction tensor and the isotropy of the Gaussian distribution. Tlusty ${ }^{54}$ followed a similar derivation to attain this result and extended the analysis to slits where the floor and ceiling are not perfectly parallel planes. Physically, the cancellation amounts to a many-body cancellation effect in an isotropic system, yielding a system with no net force due to HI. ${ }^{34} \mathrm{We}$ note that there may be interesting conformational repercussions due to incomplete cancellations at the outer "edge" of the polymer coil under a steady external force.
We continue with this analysis, however, noting the fact that the instantaneous conformation of the polymer is not isotropic. ${ }^{55,56}$ However, above we have ensemble averaged many anisotropic configurations and used the isotropy of the resulting distribution to cancel hydrodynamic interactions by symmetry. In the low-Reynolds number limit, the solvent velocity field forms instantaneously compared to the rearrangement (relaxation) time of the polymer. Therefore, this instantaneous anisotropy may be important in the hydrodynamic interaction of the system. The argument for hydrodynamic screening can be made stronger if it can be shown that the decay of the velocity magnitude is sufficient to screen hydrodynamic interactions. In this way, we can relax the preaveraging assumptions somewhat without losing analytical tractability. In the following, we will assume that the interactions have no orientational dependence. Now, the direction of the resultant velocity of bead $n$ due to HI with bead $m$ is the same as the direction of the force on bead $m$. We examine the scenario with the highest possible amount of directional cooperativity between monomer units. The preaveraged interaction tensor becomes

$$
\begin{aligned}
& \boldsymbol{H}_{n m, \mathrm{eq}}(r) \sim \\
& \int_{0}^{\infty} \frac{1}{r^{2}}\left(\frac{3}{2 \pi|n-m| L_{\mathrm{p}}^{2}}\right) \exp \left(\frac{-r^{2}}{2|n-m| L_{\mathrm{p}}^{2}}\right) r \mathrm{~d} r \boldsymbol{I}\left(1-\delta_{n m}\right)+ \\
& \boldsymbol{A}_{n} \delta_{n m}
\end{aligned}
$$

where $r=\left|\boldsymbol{r}_{n m}\right|$. Since we are concerned mainly with large length scale behavior, the local behavior described in the detailed form of $\boldsymbol{A}_{n}$ is not of importance. For simplicity, we take $\boldsymbol{A}_{n}$ to be isotropic (i.e., $\boldsymbol{A}_{n}=A_{n} \boldsymbol{I}$ ). Equation 10 then simplifies to

$$
\begin{aligned}
\boldsymbol{H}_{n m, \mathrm{eq}}= & H_{n m, \mathrm{eq}} \boldsymbol{I}= \\
& {\left[\int_{0}^{\infty} H(r) \Psi(|n-m|, r) \mathrm{d} r\left(1-\delta_{n m}\right)+A_{n} \delta_{n m}\right] \boldsymbol{I} }
\end{aligned}
$$

where $H(r)$ is the magnitude dependence of the interaction tensor $\boldsymbol{H}_{n m}$ and $\Psi(|n-m|, r)$ is still the same Gaussian bead distribution function, but it is stressed that there is no angular dependence. We now insert eq 11 into eq 6 in the place of the nonpreaveraged interaction tensor, and average over all beads $n$ to obtain a center of mass velocity

$$
\frac{1}{N} \sum_{n=1}^{N} \frac{\partial \boldsymbol{r}_{n}}{\partial t}=v_{c m}(t) \sim \frac{1}{N} \sum_{n=1}^{N} \sum_{m=1}^{N} \boldsymbol{H}_{n m, \mathrm{eq}}(r) \cdot\left(\boldsymbol{f}_{m}^{\mathrm{B}}+\boldsymbol{f}_{m}^{\mathrm{T}}+\boldsymbol{f}_{m}^{\mathrm{E}}\right)
$$

Taking the equilibrium average of eq 12 causes the Brownian and tension forces to vanish. Calling upon the isotropy of the modified interaction tensor (eq 11), the dot product can be canceled and the surviving terms are the following

$$
\left\langle v_{c m}(t)\right\rangle_{\mathrm{eq}} \sim \frac{1}{N} \sum_{n=1}^{N} \sum_{m=1}^{N} H_{n m, \mathrm{eq}}(r) \boldsymbol{f}_{m}^{\mathrm{E}}
$$

By analogy to the expression for the drag on the chain, $\xi_{\text {chain }}\langle v(t)\rangle_{e q}=N f_{m}^{\mathrm{E}}$, we can write ${ }^{57}$

$$
\begin{aligned}
& \frac{1}{\xi_{\text {chain }}} \sim \\
& \quad \frac{1}{N^{2}} \sum_{n=1}^{N} \sum_{m=1}^{N}\left(\int_{0}^{\infty} H(r) \Psi(|n-m|, r) \mathrm{d} r\left(1-\delta_{n m}\right)+A_{n} \delta_{n m}\right)
\end{aligned}
$$


Table 2. Summary of Scaling Results

\begin{tabular}{|c|c|c|c|c|c|c|c|}
\hline condition & $v$ & $H(r)$ & $g(r) \sim N / r^{\mathrm{d}}$ & $\int H(r) g(r) \mathrm{d} r$ & $\left\langle\xi_{\text {chain }}\right\rangle_{\mathrm{eq}}$ & model type & $\alpha$ \\
\hline $3 \mathrm{~d}$ ideal chain & $1 / 2$ & $1 / r$ & $1 / r$ & $N^{1 / 2}$ & $N^{1 / 2}$ & nonlocal & $\alpha>2$ \\
\hline Q2d ideal chain & $1 / 2$ & $1 / r^{2}$ & const & $\ln (N)$ & $\mathrm{N} / \ln (N)$ & nonlocal & $\alpha>2$ \\
\hline $3 \mathrm{~d}(\mathrm{SAW})$ & $3 / 5$ & $1 / r$ & $1 / r^{4 / 3}$ & $N^{2 / 5}$ & $N^{3 / 5}$ & nonlocal & $\alpha>5 / 3$ \\
\hline Q2d(SAW) & $3 / 4$ & $1 / r^{2}$ & $1 / r^{2 / 3}$ & $N^{-1 / 2}$ & $N$ & local & $\alpha>4 / 3$ \\
\hline $3 \mathrm{~d}$ rod & 1 & $1 / r$ & $1 / r^{2}$ & $\ln (N)$ & $N / \ln (N)$ & nonlocal & $\alpha>2$ \\
\hline
\end{tabular}

${ }^{a} v$ is the Flory exponent, $d$ is the dimensionality ( 2 or 3 ), and $\alpha$ describes the range of the applicability of a local model at large $N$ if $H(r) \sim 1 / r^{\alpha}$.

The second term can be written in terms of an average (scalar) mobility of a single bead. We note that the first term in the expression is not explicitly dependent on either $n$ or $m$, but only on their difference. In the long-chain limit, end effects are not important and the expression is not dependent on the position of bead $n$ along the chain. We can then write the double sum as a single sum $\sum_{\mathrm{n}=1}^{N} \sum_{\mathrm{n}=1}^{N} \Longrightarrow N \sum_{n-m=1}^{N}$. Exchanging the order of summation and integration, we obtain a direct sum of the pair probability distribution function over all beads, which is the pair correlation function. ${ }^{58}$ The expression for the drag becomes

$$
\frac{1}{\xi_{\text {chain }}} \sim \frac{1}{N}\left(\int_{L_{p}}^{L_{p} N^{v}} H(r) g(r) \mathrm{d} r+\left\langle A_{n}\right\rangle\right)
$$

where $g(r)$ is the pairwise correlation between monomers and $v$ is the Flory-Edwards exponent. We have altered the limits of integration to reflect the fact that we are most concerned with HI effects at the length scale of the coil. Also, $g(r)$ is known to decay sharply to zero for $r$ greater than the coil dimension, ${ }^{59}$ so to within a scaling approximation, the majority of the interactions are accounted for within the integral. We take the lower limit of the integral to be the smallest statistically independent step length of the polymer (a persistence length). This limit describes the effects of $\mathrm{HI}$ at the local level and does not appear (except as a prefactor) in the scaling analysis. The integral represents all of the pair-interactions between monomers through the solvent (i.e., the off-diagonal elements of the interaction tensor) from local effects (lower limit) to coil-length effects (upper limit). The second term represents the drag on an individual bead (i.e., the diagonal components of the interaction tensor). Equation 15 has been obtained by Long et al. ${ }^{50}$ though details were not given which allow one to follow the assumptions leading to this expression. Since we are concerned with long length scale and far field effects, we are concerned primarily with the behavior in the limit of large $N$. If the integral evaluated at the upper limit dominates in the limit of large $N$, the motion is cooperative and $\mathrm{HI}$ is not screened. If the integral evaluated at the upper limit vanishes in the limit of large $N$, then the motion of polymer segments can be treated as independent at a length scale smaller than $R_{\mathrm{g}}$. Therefore, a model can be devised using only local effects (i.e., $\xi_{\text {chain }} \sim N$ ) by coarsegraining the chain at a length scale larger than this hydrodynamic screening length and smaller than that of the coil. We can now use scaling assumptions ${ }^{59}$ to express the dependence of $g(r)$ on $r$, and then insert this into eq 15 to find the effect of the longranged HI.

The application of eq 15 for several case studies of solvent quality and dimensionality are summarized in Table 2. For each condition, there are two important pieces of required information: the rate of velocity decay $(H(r))$ and the pairwise correlation function of the polymer $(g(r))$. For the geometries at hand, these scalings are readily available or calculable. ${ }^{59,60}$ Evaluation of the integral in eq 15 determines the strength of the hydrodynamic interactions between the beads. In particular, the value of this integral evaluated at the upper limit reflects the importance of long-range intrachain HI. In polymer physics, screening is typically said to take place if the chain can be replaced by a series of non-hydrodynamically interacting segments such that $\xi_{\text {chain }} \sim N$. For this to occur, the integral evaluated at the upper limit must be $O(1)$ or vanish in the limit $N \rightarrow \infty$. For the three-dimensional cases, our results are in accord with known scalings. For example, for an ideal polymer (theta conditions) in $3 \mathrm{~d}$ (unconfined) the upper limit of the integral in eq 15 is the dominant contribution to the drag and we recover the well-known result that drag scales as $N^{1 / 2}$ and not as $N$. The Q2D results point to the fact that $\mathrm{HI}$ are dependent not only on the form of the velocity decay but also on the monomer distribution. It is often cited that a velocity decay alone is sufficient information to qualify a system as hydrodynamically screened, but we see in Table 2 that this is not the case. The distribution of the monomers (dependent on the solvent quality) plays a large role in the resulting dynamics of the chain. For example, consider two polymers in Q2D (i.e., with the same rate of velocity decay $H(r)$ ) which differ only in their solvent quality. For a polymer in a $\Theta$-solvent in Q2D, the velocity decay is not sufficient to prevent cooperative behavior (though the effect is only logarithmic), while for a polymer in good solvent in the same geometry the decay alone does suffice. We have shown that the magnitude decay of the velocity field alone is sufficient to cause hydrodynamic screening over equilibrium conformations of polymers in good solvents in Q2D environments.

We should note that hydrodynamic screening in confinement has been shown before. Bakajin et al. ${ }^{18}$ showed hydrodynamic screening by comparison to a model based on a hydrodynamic screening length on the order of the persistence length of the polymer. Rädler et al. ${ }^{26}$ showed hydrodynamic screening of DNA confined to two dimensions in a cationic bilayer directly next to a solid surface. In short, previous experiments have focused on the screening of hydrodynamics at length scales comparable to the persistence length. While information at these small scales is important, applications concerned with the use of confinement to control the dynamics of a single molecule require information at the scale of the coil as well. We have shown that a significant coil-scale behavioral change to freedraining occurs in slit confinements that are very close to the bulk radius of gyration of the coil.

\section{Conclusion}

We use the precision and control of microfabrication techniques, biological macromolecules, and single molecule microscopy to perform systematic experiments of DNA diffusion in slit confinement. Separate analysis of the trends with channel height and molecular weight allows interogation of different portions of confinement theories. Scalings of diffusivity with channel height do not agree well with existing theories, but the polymers here may be too short (and therefore must be confined to gaps that are too small compared to the persistence length and the thermal blob size) to allow a full comparison to the theory. Scalings with molecular weight directly show intramolecular hydrodynamic interactions in DNA solutions are screened 
when the channel height is smaller than the bulk radius of gyration. A scaling theory shows that both an orientational average and sole consideration of the algebraic decay of the hydrodynamic interactions can lead to free-draining like behavior in Q2D systems. The derivation also shows that information about the decay of the solvent velocity due to a disturbance is not sufficient to determine whether HI can be neglected at the coil's length scale, information about the spatial distribution of the hydrodynamic centers (i.e., solvent quality and spatial geometry) is needed as well.

Experimental studies of fundamental polymer physics require a significant level of detail in the results, even to test scaling predictions. Here, the use of DNA eliminates uncertainty due to molecular weight polydispersity and enables the use of direct visualization to quantify the motion of an individual polymer. The use of micro- and nano- fabrication techniques enables precise control of the channel dimensions and geometry. This increased control allows the design of experiments to test very specific hypotheses, which is ideal for fundamental studies. Furthermore, these slitlike geometries are important motifs in emerging lab-on-chip devices. ${ }^{10,61}$

Acknowledgment. We are grateful to Patrick Underhill for helpful discussions. This work was supported by the DuPontMIT Alliance, NSF Career Grant CTS-0239012, and NER Grant CTS-0304106.

\section{References and Notes}

(1) Cao, H.; Yu, Z.; Wang, J.; Tegenfeldt, J. O.; Austin, R. H.; Chen, E.; Wu, W.; Chou, S. Y. Appl. Phys. Lett. 2002, 81, 174.

(2) Mao, P.; Han, J. Lab Chip 2005, 5, 837.

(3) Reisner, W.; Morton, K. J.; Riehn, R.; Wang, Y. M.; Yu, Z.; Rosen, M.; Sturm, J. C.; Chou, S. Y.; Frey, E.; Austin, R. H. Phys. Rev. Lett. 2005, 94, 196101.

(4) Tegenfeldt, J. O.; Prinz, C.; Cao, H.; Chou, S.; Reisner, W.; Riehn, R.; Wang, Y. M.; Cox, E. C.; Sturm, J. C.; Silberzan, P.; Austin, R. H. Proc. Nat. Acad. Sci. U.S.A. 2004, 101, 10979.

(5) Chan, E. Y.; Goncalves, N. M.; Haeusler, R. A.; Hatch, A. J.; Larson, J. W.; Maletta, A. M.; Yantz, G. R.; Carstea, E. D.; Fuchs, M.; Wong, G. G.; Gullans, S. R.; Gilmanshin, R. Genome Res. 2004, 14, 1137.

(6) Randall, G. C.; Schultz, K. M.; Doyle, P. S. Lab Chip 2006, 6, 516.

(7) Riehn, R.; Lu, M.; Wang, Y.-M.; Lim, S. F.; Cox, E. C.; Austin, R. H. Proc. Natl. Acad. Sci. U.S.A. 2005, 102, 10012.

(8) Inatomi, K.; Izuo, S.; Lee, S.; Ohji, H.; Shiono, S. Microelectron. Eng. 2003, 70, 13

(9) Tegenfeldt, J. O.; Prinz, C.; Cao, H.; Huang, R. L.; Austin, R. H.; Chou, S. Y.; Cox, E. C.; Sturm, J. C. Anal. Bioanal. Chem. 2004, $378,1678$.

(10) Han, J.; Craighead, H. Science 2000, 288, 1026.

(11) Doyle, P. S.; Bibette, J.; Bancaud, A.; Viovy, J.-L. Science 2002, 295 , 2237.

(12) Turner, S. W.; Perez, A. M.; Lopez, A.; Craighead, H. G. J. Vac. Sci. Technol. B 1998, 16, 3835 .

(13) Kaji, N.; Tezuka, Y.; Takamura, Y.; Ueda, M.; Nishimoto, T.; Nakanishi, H.; Horiike, Y.; Baba, Y. Anal. Chem. 2004, 76, 15.

(14) Pennathur, S.; Santiago, J. G. Anal. Chem. 2005, 77, 6772.

(15) Pennathur, S.; Santiago, J. G. Anal. Chem. 2005, 77, 6782.

(16) Bakajin, O.; Duke, T. A. J.; Tegenfeldt, J.; Chou, C.-F.; Chan, S. S.; Austin, R. H.; Cox, E. C. Anal. Chem. 2001, 73, 6053.

(17) Chen, Y.-L.; Graham, M. D.; de Pablo, J. J.; Randall, G. C.; Gupta, M.; Doyle, P. S. Phys. Rev. E 2004, 70, 060901(R).

(18) Bakajin, O. B.; Duke, T. A. J.; Chou, C. F.; Chan, S. S.; Austin, R. H.; Cox, E. C. Phys. Rev. Lett. 1998, 80, 2737.
(19) Brochard, F.; de Gennes, P. G. J. Chem. Phys. 1977, 67, 52.

(20) Burkhardt, T. W. J. Phys. A: Math. Gen. 1997, 30, L167.

(21) Daoud, M.; de Gennes, P. G. J. Phys. (Paris) 38, 85.

(22) Deen, W. M. AIChE J. 1987, 33, 1409.

(23) Odijk, T. Macromolecules 1983, 16, 1340.

(24) Wall, F. T.; Seitz, W. A.; Chin, J. C.; de Gennes, P. G. Proc. Natl. Acad. Sci. U.S.A. 1978, 75, 2069.

(25) Maier, B.; Rädler, J. O. Macromolecules 2000, 33, 7185.

(26) Maier, B.; Rädler, J. O. Phys. Rev. Lett. 1999, 82, 1911.

(27) Jendrejack, R. M.; Schwartz, D. C.; Graham, M. D.; de Pablo, J. J. J. Chem. Phys. 2003, 119, 1165.

(28) Usta, O. B.; Ladd, A. J. C.; Butler, J. E. J. Chem. Phys. 2005, 122, 094902.

(29) Liron, N.; Mochon, S. J. Eng. Math. 1976, 10, 287.

(30) Flory, P. J. Principles of Polymer Chemistry, Cornell University Press: Ithaca, NY, 1953.

(31) Choi, M. C.; Santangelo, C. D.; Pelletier, O.; Kim, J. H.; Kwon, S. Y.; Wen, Z.; Li, Y.; Pincus, P. A.; Safinya, C. R.; Kim, M. W. Macromolecules 2005, 38, 9882.

(32) Köster, S.; Steinhauser, D.; Pfohl1, T. J. Phys.: Condens. Matter 2005, $17, \mathrm{~S} 4091$.

(33) Morse, D. C. Macromolecules 1998, 31, 7044.

(34) Alvarez, A.; Soto, R. Phys. Fluids 2005, 17, 093103.

(35) Diamant, H.; Cui, B.; Lin, B.; Rice, S. A. J. Phys.: Condens. Matter 2005, 17, S2787.

(36) Lin, Y.-W.; Huang, C.-C.; Chang, H.-T. Anal. Bioanal. Chem. 376.

(37) Cui, B.; Diamant, H.; Lin, B.; Rice, S. A. Phys. Rev. Lett. 2004, 92, 258301.

(38) Perkins, T. Exploring Polymer Dynamics with Single DNA Molecules. Ph.D. Thesis, Stanford University, 1997.

(39) Smith, D. E.; Perkins, T. T.; Chu, S. Macromolecules 1996, 29, 1372

(40) Stellwagen, N. C.; Gelfi, C.; Righetti, P. G. Biophys. J. 2000, 54 , 137.

(41) Randall, G. Single Molecule Analysis of DNA Electrophoresis in Microdevices. Ph.D. Thesis, Massachusetts Institute of Technology, 2006.

(42) Haile, J. M. Molecular Dynamics Simulation: Elementary Methods; Wiley-Interscience: New York, 1992.

(43) Goodman, A.; Tseng, Y.; Wirtz, D. J. Mol. Biol. 2002, 323, 199.

(44) Marko, J. F.; Siggia, E. D. Macromolecules 1995, 28, 8759.

(45) Rubenstein, M.; Colby, R. H. Polymer Physics; Oxford University Press: New York 2003.

(46) Hsu, H.-P.; Grassberger, P. J. Chem. Phys. 2004, 120, 2034.

(47) Harden, J. L.; Doi, M. J. Phys. Chem. 1992, 96, 4046.

(48) Dechadilok, P.; Deen, W. M. Ind. Eng. Chem., in press

(49) Happel, J.; Brenner, H. Low Reynolds number hydrodynamics; Kluwer: Boston, MA, 1983.

(50) Long, D.; Viovy, J.-L.; Ajdari, A. J. Phys.: Condens. Matter 1996, $8,9471$.

(51) Doi, M.; Edwards, S. F. The Theory of Polymer Dynamics, Oxford University Press: New York, 1986.

(52) Underhill, P. T.; Doyle, P. S. J. Rheol. 2005, 49, 963.

(53) Woo, N. J.; Shaqfeh, E. S. G.; Khomami, B. J. Rheol. 2004, 48, 281.

(54) Tlusty, T. Macromolecules 2006, 39, 3927.

(55) van Vliet, J. H.; ten Brinke, G. J. Chem. Phys. 1990, 93, 1436.

(56) Haber, C.; Ruiz, S. A.; Wirtz, D. Proc. Nat. Acad. Sci. U.S.A. 2000, 97, 10792.

(57) Bird, R. B.; Curtiss, C. F.; Armstrong, R. C.; Hassager, O. Dynamics of Polymeric Liquids, Volume 2 kinetic Theory, 2nd ed.; WileyInterscience, New York, 1987.

(58) Graessley, W. M. Polymeric Liquids and Networks: Structure and Properites; Garland Science: New York 2004.

(59) de Gennes, P.-G. Scaling Concepts in Polymer Physics, Cornell University Press: Ithaca, NY, 1979.

(60) Pozrikidis, C. Intoduction to Theoretical and Computational Fluid Dynamics; Oxford University Press: New York, 1997.

(61) Wang, Y.-C.; Choi, M. H.; Han, J. Anal. Chem. 2004, 76, 4426.

(62) Savin, T.; Doyle, P. S. Biophys. J. 2005, 88, 623.

\section{MA061047T}

